\title{
O Impacto do Temperamento Infantil, da Responsividade e das Práticas Educativas Maternas nos Problemas de Externalização e na Competência Social da Criança
}

\author{
The Impact of Infant Temperament, Responsiveness and Maternal Childrearing Practices \\ on Children Externalizing Behavior Problems and Social Competence
}

\author{
Patrícia Alvarenga* \& Cesar A. Piccinini \\ Universidade Federal do Rio Grande do Sul, Porto Alegre, Brasil
}

\begin{abstract}
Resumo
O estudo investigou o impacto do temperamento infantil, da responsividade materna e das práticas educativas maternas, nos problemas de externalização e na competência social de crianças. Participaram 23 díades mãecriança de diferentes níveis socioeconômicos. No $3^{\circ}$ mês de vida do bebê avaliou-se o temperamento da criança e a responsividade materna. No $30^{\circ}$ mês de vida da criança foram investigadas as práticas educativas maternas, os problemas de externalização e a competência social das crianças. Análise de regressão linear múltipla revelou que somente as práticas educativas foram fatores significativos para explicar a variância nos problemas de externalização e na competência social. Discute-se a relevância das práticas parentais e as limitações da avaliação do temperamento e responsividade materna como preditores para a compreensão do desenvolvimento social. Palavras-chave: Temperamento; responsividade; práticas educativas parentais.
\end{abstract}

\begin{abstract}
The study aimed at investigating the impact of children temperament, maternal responsiveness and maternal childrearing practices on children externalizing behavior problems and social competence. The study involved 23 child-mother dyads from different socio-economical backgrounds. In the baby's third month after birth, child temperament and maternal responsiveness were evaluated. In the child's thirteenth month after birth, maternal childrearing practices, externalizing behavior problems and child social competence were investigated. Multiple regression analysis revealed that only maternal childrearing practices were significant to explain the variance in the externalizing behaviors and social competence. The work discusses the relevance of parental practices and the limitations of the evaluation of temperament and maternal responsiveness as predictors for the comprehension of social development.

Keywords: Temperament; responsiveness; childrearing practices.
\end{abstract}

O desenvolvimento social na infância tem sido objeto de estudo de inúmeras investigações devido a suas implicações para o funcionamento do indivíduo em etapas posteriores do desenvolvimento. Neste contexto, os conceitos de competência social e de problemas de externalização têm sido utilizados para fazer referência a diferentes padrões de desenvolvimento social nos primeiros anos de vida.

Os problemas de externalização, que envolvem comportamentos como agressividade, impulsividade e comportamento desafiador, têm sido amplamente investigados na

\footnotetext{
*Endereço para correspondência: Departamento de Psicologia, Faculdade de Filosofia e Ciências Humanas, Universidade Federal da Bahia, Rua Aristidis Novis, 197, Federação, Salvador, BA, 40210-730. E-mail: palvarenga66@gmail.com

Este estudo foi baseado na tese de doutorado de Patrícia Alvarenga, realizada sob a supervisão de Cesar Piccinini, apresentada no Programa de Pós-Graduação em Psicologia do Desenvolvimento da Universidade Federal do Rio Grande do Sul, Porto Alegre - RS. Agradecemos a colaboração de Ana Paula Vieira, Ana Paula Sabocinski, Airana Fidelis da Luz Moura e Luciana Ouriques durante a análise dos dados.
}

etapa do desenvolvimento que compreende o final da infância e a adolescência (Achenbach \& Howell, 1993; Weisz, Chaiyaist, Weiss, Eastman \& Jackson, 1995). No entanto, apenas recentemente passou-se a considerar a relevância clínica dos indicadores de externalização apresentados por crianças com menos de seis anos como preditores de possíveis dificuldades futuras (Keenan \& Shaw, 1998; Patterson, DeGarmo, \& Knutson, 2000).

Há evidências de que as primeiras manifestações dos problemas de externalização podem ser detectadas precocemente, aproximadamente entre os 18 e os 24 meses (Crockenberg \& Litman, 1990; Keenan \& Shaw, 1997). Nessa idade a criança já apresentaria tendência a agredir os pais ou pares, a destruir objetos e à conduta desafiadora. Além disto, estudos longitudinais que acompanham crianças entre o período pré-escolar e o final da infância oferecem evidências bastante consistentes da estabilidade dos indicadores de externalização (Denham, Workman, Cole, Weissbrod \& Zahn-Waxler, 2000; Shaw, Owens, Giovanelli \& Winslow, 
2001). De modo semelhante, indicadores de competência social também já podem ser avaliados nesta etapa do desenvolvimento. A competência social envolve uma série de habilidades que variam entre as culturas e que permitem ao indivíduo estabelecer relações positivas com os outros (Durkin, 1995).

O termo competência social é muitas vezes utilizado na literatura como sinônimo do conceito de habilidades sociais. No entanto, Del Prette e Del Prette (1999) assinalam que, embora sejam conceitos intimamente relacionados, há diferenças claras que devem ser consideradas. O conceito de habilidades sociais compõe-se de elementos comportamentais como expressão de sentimentos, empatia, antecipação de conseqüências, entre outros. Por outro lado, a competência social se verifica na medida em que o indivíduo é capaz de integrar esse conjunto de habilidades às demandas que emergem nas relações sociais, observando as exigências éticas impostas pela cultura. A competência social seria, portanto, a capacidade de a pessoa apresentar um comportamento que possa atingir os objetivos de uma situação interpessoal, mantendo uma relação de equilíbrio de poder e de trocas positivas com o interlocutor (Castro, Melo \& Silvares, 2003). Desta forma, o conceito de competência social abrange o de habilidades sociais, e acrescenta a noção de adequação do comportamento às demandas do contexto em que ele ocorre. A competência social constitui-se, portanto, em um conjunto de habilidades, do qual fazem parte a empatia e o altruísmo (também referidos na literatura como comportamentos pró-sociais), a assertividade e a cooperação, entre outras (Cecconello \& Koller, 2000; Crockenberg \& Litman, 1990).

Durante a infância a competência social se manifesta, essencialmente, na interação da criança com seus pais e com o grupo de companheiros, e envolve habilidades como a empatia, a assertividade (Falcone, 2001) e a obediência, considerada um preditor da cooperação (Crockenberg \& Litman, 1990; Maccoby, 1966). Além desses indicadores específicos da competência social, alguns autores argumentam que a habilidade da criança de engajar-se em interações sociais de forma positiva com os pais, incluindo gestos, vocalizações, olhares e demonstrações de afeto positivo, são importantes sinalizadores de um desenvolvimento social apropriado (Landry, Smith, Swank, Assel \& Vellet, 2001).

Patterson, Reid e Dishion (1992) desenvolveram um modelo para explicar o início do processo de socialização, que ocorre no contex to familiar. De acordo com este modelo, o conceito chave para a compreensão do desenvolvimento da competência social ou de problemas de externalização é o de contingência das práticas educativas parentais. Este conceito faz referência ao fato de os pais comportarem-se efetiva e consistentemente no sentido de reduzir comportamentos considerados inadequados, e de estimular a ocorrência de comportamentos socialmente adequados. As definições de comportamento adequado e comportamento inadequado remetem a comportamentos considerados aversivos e, portanto, passíveis de punição pelo grupo social (comportamentos inadequados), e comportamentos que constituem reforçadores positivos e que costumam ser também reforçados pelo grupo social (comportamentos adequados). Estas definições dependem também, em nível operacional, de uma série de variáveis inerentes ao contexto social e cultural em que o comportamento ocorre.

O resultado de práticas educativas contingentes seria o de fornecer à criança, entre outras coisas, previsibilidade e, portanto, organização ao seu repertório comportamental. Por outro lado, práticas parentais não contingentes levariam ao desenvolvimento de um repertório pobre em competência social e aos problemas de externalização. Para Patterson et al. (1992), os membros da família podem treinar diretamente comportamentos socialmente inadequados na criança, configurando o que os autores chamam de ciclo familiar coercitivo. Os pais, em geral, não são contingentes no uso de reforçadores positivos e fracassam no uso efetivo de técnicas disciplinares para enfraquecer os comportamentos inadequados. Além disso, essas famílias são caracterizadas por uma disciplina severa e inconsistente (Fox, Platz \& Bentley, 1995; Pettit, Bates \& Dodge, 1997).

Outra perspectiva sobre a relação entre as práticas educativas parentais e o comportamento infantil foi oferecida por Hoffman $(1979,1994)$. De acordo com este autor as estratégias indutivas (explicações, negociações), caracterizam-se por atingir o objetivo disciplinar indicando para a criança as conseqüências do seu comportamento para as outras pessoas e chamando sua atenção para os aspectos lógicos da situação. Isso propicia à criança a compreensão das implicações de suas ações e, portanto, dos motivos que justificam a necessidade de mudança no seu comportamento. Desta forma, a criança desenvolve certa autonomia para utilizar esse tipo de informação para controlar seu próprio comportamento. Já as estratégias de força coercitiva que se caracterizam pela aplicação direta da força, incluindo punição física, privação de privilégios e afeto ou pelo uso de ameaças, fazem com que a criança controle seu comportamento somente em função das reações punitivas dos pais Além disso, elas produzem emoções intensas tais como medo, raiva e ansiedade, que tendem a reduzir ainda mais a possibilidade de a criança compreender a situação e a necessidade de modificação de comportamento. Ou seja, o controle do comportamento da criança tenderá a depender de intervenções externas porque ela não adquire a capacidade de compreender as implicações de suas ações (Hoffman, 1979, 1994). Nesse sentido, além da contingência das práticas parentais, o uso mais freqüente de práticas indutivas estaria associado a maior capacidade de auto-regulação por parte da criança, e, portanto, a maior competência social. De modo semelhante o uso freqüente de práticas coercitivas estaria associado a menor capacidade de auto-regulação e aos problemas de comportamento.

Vários estudos têm investigado o papel das práticas educativas parentais no desenvolvimento social (Eddy, Leve \& Fagot, 2001; Patterson et al., 1992, 2000). A maioria dos estudos confirma a importância do uso de práticas não-coercitivas (ex. explicações, negociações), do reforçamento positivo contingente e do envolvimento positivo dos pais para a com- 
petência social da criança (Arsenio \& Overton, 2004; Raver, 1996). Quanto aos problemas de externalização, a maior parte dos estudos converge para uma relação entre o uso predominante de práticas de caráter coercitivo e estes problemas comportamentais (Alvarenga \& Piccinini, 2001; Bolsoni-Silva \& Marturano, 2002; McLoyd \& Smith, 2002). Partindo da perspectiva de Patterson et al. (1992), é possível que estas práticas, embora coercitivas, sejam pouco contingentes, e, portanto, ineficazes para reduzir comportamentos inadequados. Por outro lado, algumas pesquisas indicam que, além da coerção, outros aspectos como a intrusividade, definida como a tendência ao controle exagerado do comportamento da criança (Dumas \& LaFreniere, 1993; Oliveira, Frizzo \& Marin, 2000), a autorização de autonomia (Denham, Renwick \& Holt, 1991) e o uso do controle assertivo (Crockenberg \& Litman, 1990; Patterson et al., 1992), também são variáveis relevantes para o entendimento destas relações.

Alguns estudos indicam a necessidade de que sejam conduzidas investigações que permitam traçar as origens do processo de interação entre as práticas educativas parentais e o desenvolvimento social infantil (Cohen \& Brook, 1998; Keenan \& Shaw, 1998). Para muitos pesquisadores o nível de responsividade dos pais ao comportamento dos seus bebês seria um forte preditor do tipo e da eficácia das práticas educativas que serão empregadas no futuro (Keenan \& Shaw, 1998; Patterson et al., 2000). Pais menos hábeis e com menor disponibilidade emocional para perceber os sinais de seus bebês e responder adequadamente a eles, tenderiam a apresentar maior dificuldade de regular o comportamento de seus filhos no futuro, empregando, com maior frequiência, práticas coercitivas, severas e pouco eficazes, enquanto pais mais sensíveis ao comportamento de seus bebês, tenderiam a ser mais hábeis na regulação do comportamento dos filhos em etapas posteriores do desenvolvimento. $\mathrm{O}$ conceito de responsividade pode ser definido como atenção e percepção consistentes, interpretação acurada e resposta contingente e apropriada aos sinais da criança (De Wolff \& Ijzendoorn, 1997; van den Boom, 1994). Essa característica dos pais favoreceria a ocorrência de interações sincrônicas e mutuamente recompensadoras entre os elementos da díade.

Por outro lado, examinando as características e contribuições da criança para a interação, o temperamento infantil também é mencionado, tanto como um preditor precoce da vulnerabilidade da criança para os problemas de externalização (Guerin, Gottfried \& Thomas, 1997) quanto como um preditor da quantidade e do tipo de cuidado que os pais irão dispensar a ela (van den Boom, 1994). O temperamento pode ser definido como um padrão inato e estável de humor e de reação, que refere-se a diferenças constitucionais na reatividade emocional, motora e atencional (Sanson, Hemphill \& Smart, 2002; Schwebel \& Plumert, 1999). Embora o temperamento se diferencie de outros atributos comportamentais por seu caráter inato, os pesquisadores afirmam que ele pode ser modificado pela maturação e pelo ambiente (Carey \& McDevitt, 1995; Rothbart, Ahadi, Hershey \& Fisher, 2001).
Existem estudos norte-americanos e europeus que analisam o papel da interação de alguns desses fatores nos problemas de externalização. Contudo, em alguns estudos a avaliação do temperamento infantil e da responsividade materna foi relativamente tardia (após o primeiro ano de vida da criança) (Keenan \& Shaw, 1998; Shaw et al., 2001), e em outros foram utilizadas amostras de crianças que já apresentavam problemas de externalização, sendo que esses fatores foram avaliados com base em dados retrospectivos (Patterson et al., 2000).

Assim, o objetivo do presente estudo foi o de investigar o impacto do temperamento infantil e da responsividade materna, avaliados aos três meses de vida do bebê, e das práticas educativas maternas, em indicadores iniciais de problemas de externalização e competência social de crianças aos 30 meses de vida. A partir das perspectivas teóricas de Patterson et al. (1992) e Hoffman (1979), para o presente estudo, optou-se pela utilização de uma terminologia que contemplasse de forma mais ampla as práticas educativas parentais relacionadas ao desenvolvimento da competência social e dos problemas de externalização, sem privilegiar apenas o aspecto da presença ou ausência de coerção. Portanto, o termo práticas educativas facilitadoras do desenvolvimento social, será utilizado para designar as estratégias como a assertividade, a sensibilidade o envolvimento positivo e as orientações maternas utilizadas na regulação do comportamento da criança. Já o termo práticas educativas nãofacilitadoras do desenvolvimento social será utilizado para designar as estratégias coercitivas, intrusivas, ambíguas ou permissivas de controle.

A hipótese era a de que a baixa responsividade materna e o temperamento difícil da criança estariam relacionados a práticas não-facilitadoras do desenvolvimento social e aos problemas de externalização na criança aos 30 meses, enquanto a alta responsividade materna e o temperamento fácil estariam relacionados a práticas facilitadoras do desenvolvimento, e à competência social.

\section{Participantes}

\section{Método}

Participaram deste estudo 23 díades mãe-criança de diferentes níveis socioeconômicos. As crianças (13 meninos e 10 meninas) eram saudáveis e nascidas a termo. Todas as mães eram primíparas, com idade média de 24,39 anos $(D P=5,88)$ e residiam com seus maridos ou companheiros na região metropolitana de Porto Alegre.

A amostra foi selecionada, com base nos critérios descritos acima, dentre os participantes do "Estudo Longitudinal de Porto Alegre: Da Gestação à Escola" (Piccinini, Tudge, Lopes \& Sperb, 1998). Este estudo iniciou acompanhando 81 gestantes, que não apresentavam intercorrências clínicas fossem com elas mesmas ou com o bebê, que era seu primeiro filho. Os maridos ou companheiros, também foram convidados a participar do estudo caso residissem juntos em situação matrimonial. Os participantes representavam várias configurações familiares (nucleares, monoparentais ou re-casados), de dife- 
rentes idades (adultos e adolescentes) e com escolaridade e níveis socioeconômicos variados. O estudo envolveu várias fases de coleta de dados desde a gestação até o sétimo ano das crianças (gestação, $3^{\circ}, 8^{\circ}, 12^{\circ}, 18^{\circ}, 24^{\circ}, 30^{\circ}$ mês e $6^{\circ}$ e $7^{\circ}$ ano de vida da criança) Teve por objetivo investigar tanto os aspectos subjetivos e comportamentais das interações iniciais pai-mãe-bebê, assim como o impacto de fatores iniciais do desenvolvimento nas interações familiares, no comportamento social de pré-escolares e na transição para o ensino fundamental. O convite inicial para participar do estudo ocorreu quando a gestante fazia pré-natal em hospitais da rede pública da cidade de Porto Alegre (51,2\%), nas unidades sanitárias de saúde do mesmo município (7,3\%), por meio de anúncios em veículos de comunicação (26,8\%) epor indicação (14,6\%). A fora os dados sociodemográficos coletados na gestação, o presente estudo considerou apenas dados obtidos no $3^{\circ}$ e no $30^{\circ}$ mês de vida da criança.

O nível socioeconômico das famílias foi avaliado a partir dos critérios propostos por Hollingshead (1975), adaptados por Tudge e Frizzo (2002). Esse índice foi calculado, para cada indivíduo, somando-se o nível educacional multipli- cado por três com o prestígio ocupacional multiplicado por cinco. No caso de famílias nucleares, os escores individuais para cada um dos cônjuges foram somados e foi calculada uma média simples que representa o status socioeconômico da família. Essa classificação revelou uma distribuição relativamente equilibrada na amostra. A Tabela 1 apresenta as características demográficas dos participantes do estudo.

\section{Procedimentos}

Quando os bebês complataram três meses de vida, foram realizadas a Entrevista sobre características do temperamento do bebê- $3^{a}$ mês e a Observação da interação familiar $-3^{o}$ mês para a avaliação da responsabilidade materna, durante uma visita domiciliar. As entrevistas foram gravadas em audiotape e as observações foram filmadas. Aos 30 meses de idade das crianças, as famílias foram novamente visitadas em suas residências para a realização da Observação da interação familiar $-30^{\circ}$ mês, que foi utilizada para a avaliação das práticas educativas maternas e dos indicadores de problemas de externalização e de competência social nas crianças. A sessão de observação foi filmada.

Tabela 1

Características Demográficas dos Participantes ( $n=23$ )

\begin{tabular}{lc}
\hline Sexo da criança & \\
Feminino & $10(43,5 \%)$ \\
Masculino & $13(56,5 \%)$ \\
Idade da mãe no momento da gestação & $\mathrm{M}=24,39$ \\
& $\mathrm{DP}=5,88)$ \\
Idade do pai no momento da gestação & $\mathrm{M}=27,57$ \\
& $\mathrm{DP}=7,22)$ \\
Escolaridade da mãe & \\
Ensino fundamental incompleto & $1(4,3 \%)$ \\
Ensino fundamental completo & $3(13 \%)$ \\
Ensino médio incompleto & $3(13 \%)$ \\
Ensino médio completo 8 & $(34,8 \%)$ \\
Ensino superior incompleto & $5(21,7 \%)$ \\
Ensino superior completo & $3(13 \%)$ \\
Escolaridade do pai: & \\
Ensino fundamental incompleto & $5(21,7 \%)$ \\
Ensino fundamental completo & $1(4,3 \%)$ \\
Ensino médio incompleto & $2(8,7 \%)$ \\
Ensino médio completo 7 & $(30,4 \%)$ \\
Ensino superior incompleto & $2(8,7 \%)$ \\
Ensino superior completo & $5(21,7 \%)$ \\
Pós-graduação & $1(4,3 \%)$ \\
Estado civil da mãe & \\
Casada & $16(69,6 \%)$ \\
União estável & $7(30,4 \%)$ \\
Nível socioeconômico da família & \\
1- Bollingshead) & $5(26,1 \%)$ \\
2- Médio-baixo & $5(21,7 \%)$ \\
4- Médio & $3(13 \%)$ \\
5- Alto & $4(17,4 \%)$ \\
\hline & $5(21,7 \%)$ \\
\hline
\end{tabular}




\section{Instrumentos}

1. Entrevista de Dados Demográficos do Casal (Grupo de Interação Social, Desenvolvimento e Psicopatologia [GIDEP], 1998): essa ficha visou coletar dados como idade da gestante e do companheiro, escolaridade, profissão, estado civil, religião, tempo de trabalho, etnia e moradores da casa.

2. Observação da Interação Familiar - $3^{\circ}$ mês (GIDEP, 1999a): Foi solicitado à mãe que agisse livremente com seu bebê, como fazia normalmente quando estava com ele. A análise da responsividade materna foi realizada com base nas propostas de Isabella, Belsky e Von Eye (1989), van den Boom (1994) e Wendland-Carro, Piccinini e Millar (1999). Inicialmente, seis minutos do episódio de interação mãe-bebê (três iniciais e três finais) foram divididos em intervalos de 12 segundos. Nos primeiros seis segundos foram registrados os comportamentos do bebê em três categorias distintas: sorri, vocaliza, chora/choraminga. As respostas maternas a esses comportamentos foram registradas no mesmo intervalo e no intervalo seguinte em sete categorias distintas: interpreta/fala pelo bebê, fala para o bebê, sorri para o bebê, pega no colo/embala/aconchega, acaricia/beija, toca/estimula fisicamente ou com objeto e oferece bico/seio/mamadeira. Em uma segunda etapa da análise as três categorias de comportamentos do bebê e as sete categorias de comportamentos da mãe foram combinadas formando seqüências responsivas considerando-se os critérios de contingência e adequação da resposta materna. O critério de contingência das respostas maternas foi avaliado com base no tempo (respostas maternas que ocorreram até doze segundos após o comportamento do bebê). A adequação das respostas maternas foi avaliada com base no tipo de resposta apresentado pela mãe. Foram consideradas como respostas adequadas aos comportamentos do bebê, comportamentos maternos facilitadores da interação, ou seja, todos os comportamentos maternos expressos nas sete categorias analisadas. Desta forma, derivaram-se 21 seqüências responsivas e para cada uma delas foi calculado o percentual de responsividade materna dividindo-se o número de ocorrências naquela seqüência pelo número de comportamentos do bebê em questão. Na última etapa da análise foram calculadas as médias dos percentuais de cada grupo de sete seqüências, obtendo-se assim, os percentuais médios de responsividade materna ao sorriso, às vocalizações e ao choro do bebê. A média desses três percentuais foi utilizada como o indicador geral da responsividade. A codificação dos vídeos foi realizada por dois codificadores independentes, após extenso treinamento usando vídeos de outros estudos. A fidedignidade entre os codificadores foi estabelecida utilizando-se 20 vídeos de interação mãe-bebê do estudo longitudinal com as mesmas características dos vídeos utilizados no presente estudo. Para as sete categorias de comportamentos maternos, o coeficiente Kappa variou de $K=0,70$ a $K=0,93(M=0,82)$; para as três categorias de comportamentos infantis, variou de $K=0,71$ a $K=0,91$ $(M=0,81)$, atingindo média geral de $K=0,82$.
3. Entrevista sobre Características do Temperamento do Bebê- $3^{\circ}$ $m e \hat{s}$ (GIDEP, 1999b): A entrevista foi elaborada com base nas dimensões propostas por Seifer, Sameroff, Barrett e Krafchuk (1994), e Thomas e Chess (1977). Foram investigados o nível de atividade física, o ritmo, a reação da criança a novas situações, a adaptabilidade, a intensidade da reação emocional e o humor da criança. Estas seis dimensões foram selecionadas por serem as mais freqüentemente citadas na literatura, e também por serem utilizadas no modelo de Thomas e Chess (1977) para a caracterização do tipo de temperamento infantil. As quatro primeiras dimensões foram investigadas em nove situações do dia a dia do bebê: sono, alimentação, vestir-se, banho, troca de fraldas, mudanças na rotina, pessoas estranhas, lugares estranhos e festas. A intensidade da reação emocional e o humor da criança foram examinados globalmente, sem considerar situações específicas. A avaliação das características de temperamento foi realizada por meio da classificação das respostas das mães (para cada situação em cada uma das dimensões) em uma escala de cinco pontos, na qual o valor um designava características de temperamento fácil/adaptável e o valor cinco referia-se a características de temperamento difícil/adaptação lenta. A consistência interna da escala atingiu 0,74 (coeficiente alpha de Chronbach). Para cada uma das seis dimensões foi calculada a média aritmética. As médias nas seis dimensões foram somadas para formar o escore bruto de temperamento. O escore total foi estabelecido dividindo-se o resultado da soma das médias nas seis dimensões por seis (número de dimensões avaliadas). Desta forma, o escore final de temperamento de cada criança poderia variar de um (temperamento fácil/adaptável) a cinco (temperamento difícil/adaptação lenta). A classificação das respostas das mães foi realizada por dois codificadores independentes, após extenso treinamento no uso do protocolo de codificação. A fidedignidade entre os codificadores foi estabelecida em dez entrevistas. O Teste de Kendall W foi calculado separadamente para cada uma das situações nas seis dimensões avaliadas e variou de 0,77 a $1,0(M=0,92)$.

4. Observação da interação familiar $-30^{\circ}$ mês (GIDEP, 2001): As famílias foram filmadas durante um almoço. O contex to de alimentação foi escolhido por ser referido na literatura como um tipo de situação durante o qual oportunidades de as mães regularem o comportamento das crianças utilizando práticas educativas seriam freqüentes (Crockenberg \& Litman, 1990). O pai da criança e outros membros da família que costumavam participar das refeições, também participaram da filmagem. Para fins de análise foram considerados apenas os momentos de interação da mãe com a criança durante o almoço, a partir do momento em que a criança estava sentada à mesa. Foram selecionados os 12 minutos iniciais da filmagem para análise devido ao curto intervalo de tempo que algumas das crianças permaneceram à mesa. Os 12 minutos selecionados foram divididos em 24 intervalos de 30 segundos durante os quais foram codificadas as práticas educativas da mãe e os comportamentos da criança. A estrutura de categorias utilizada para a análise da interação foi elaborada com base nos trabalhos de 
Crockenberg e Litman (1990), Eddy et al. (2001), Landry et al. (2001), Paley, Cox e Kanoy (2001) e Patterson et al. (1992). As práticas educativas maternas foram codificadas em oito categorias distintas. As categorias orientação, sensibilidade, envolvimento positivo, e controle assertivo foram agrupadas e denominadas práticas educativas facilitadoras do desenvolvimento social, por referirem-se a práticas que não envolvem o uso da coerção e que estão relacionadas ao desenvolvimento da competência social. As categorias controle ambiguo, controle coercitivo, intrusividade e permissividade foram agrupadas como práticas educativas não-facilitadoras do desenvolvimento social, por referirem-se a práticas que envolvem o uso da coerção, ou que são referidas pela literatura como pouco eficazes para o processo de socialização, estando relacionadas ao desenvolvimento de problemas de externalização e de problemas de comportamento em geral. Os comportamentos da criança foram avaliados através de seis categorias distintas. As categorias obediência, assertividade, envolvimento positivo foram agrupadas como indicadores de competência social, por referirem-se a aspectos da conduta infantil citados na literatura como indicadores positivos do processo de socialização nesta etapa do desenvolvimento. As categorias inadequação, desobediência passiva e negativismo foram agrupadas como indicadores de problemas de externalização, por serem referidas na literatura como comportamentos que podem ser considerados preditores precoces dos problemas de externalização e da conduta anti-social. A codificação dos vídeos foi realizada por dois codificadores independentes, extensamente treinados e a fidedignidade foi estabelecida em dez vídeos do estudo longitudinal com as mesmas características dos vídeos utilizados no presente estudo. Para as oito categorias de práticas educativas o valor de Kappa variou de $K=0,66$ a $K=0,93(M=0,85)$. Para as seis categorias de comportamentos infantis, o coeficiente variou de $K=0,74$ a $K=0,93(M=0,85)$.

Tabela 2

Correlações entre as Práticas Educativas Maternas e os Indicadores de Competência Social e de Problemas de Externalização (n=23)

\begin{tabular}{|c|c|c|c|c|c|c|c|c|c|}
\hline \multicolumn{10}{|c|}{ Comportamento infantil } \\
\hline \multicolumn{10}{|c|}{ Externalização $\quad$ Competência social } \\
\hline \multicolumn{10}{|l|}{ Práticas educativas } \\
\hline \multicolumn{10}{|l|}{ Práticas facilitadoras } \\
\hline Orientação & $0,44^{*}$ & $0,47 *$ & $\mathrm{O}, \mathrm{OO}$ & 0,39 & 0,22 & 0,31 & $0,43^{*}$ & $0,42 *$ & $0,65 * *$ \\
\hline Sensibilidade & $0,50^{*}$ & $0,43^{*}$ & $-0,19$ & $-0,13$ & 0,08 & $-0,22$ & 0,38 & $-0,06$ & 0,34 \\
\hline Envolvimento positivo & 0,04 & $-0,03$ & $0,80^{* * *}$ & 0,20 & $-0,09$ & 0,04 & $0,54 * *$ & 0,06 & 0,36 \\
\hline Controle assertivo & $0,78^{* *}$ & 0,21 & $-0,04$ & 0,29 & $0,62^{* * *}$ & $-0,22$ & 0,41 & $0,46 *$ & $0,58 * *$ \\
\hline \multicolumn{10}{|l|}{ Práticas não-facilitadoras } \\
\hline Controle ambíguo & 0,37 & $-0,20$ & $-0,13$ & $0,51^{*}$ & 0,23 & $-0,20$ & $-0,05$ & 0,41 & 0,24 \\
\hline Controle coercitivo & $0,47 *$ & $-0,01$ & 0,06 & 0,38 & 0,36 & $-0,04$ & 0,20 & $0,44^{*}$ & 0,38 \\
\hline Intrusividade & 0,29 & $-0,04$ & $-0,37$ & 0,27 & 0,28 & $-0,11$ & $-0,16$ & 0,07 & 0,25 \\
\hline Permissividade & 0,29 & $-0,03$ & $-0,35$ & 0,28 & 0,35 & $-0,08$ & $-0,11$ & 0,35 & 0,22 \\
\hline Total práticas facilitadoras & $0,69^{* * *}$ & $0,47 *$ & 0,16 & 0,17 & 0,23 & $-0,00$ & $0,68 * *$ & 0,23 & $0,71 * *$ \\
\hline Total práticas não-facilitadoras & $0,53 * *$ & $-0,02$ & $-0,02$ & $0,45^{*}$ & $0,41 *$ & $-0,08$ & 0,17 & $0,42^{*}$ & $0,50^{*}$ \\
\hline Total de práticas maternas & $0,75^{* * *}$ & 0,38 & 0,16 & 0,33 & 0,31 & $-0,03$ & $0,65 * *$ & $0,74^{* *}$ & 0,36 \\
\hline
\end{tabular}

\section{Resultados}

Relações entre o Temperamento e a Responsividade Materna no $3^{\circ}$ Mês e a Competência Social e os Problemas de Externalização no $30^{\circ}$ Mês de Vida

O Teste de Correlação de Spearman revelou correlações negativas entre a intensidade da reação emocionale o envolvimento positivo $(r=-0,51 ; p<0,05)$ e novamente a intensidade emocional $\mathrm{e}$ o negativismo $(r=-0,46 ; p<0,05)$, mostrando que bebês que expressavam suas emoções de modo muito intenso aos três meses de vida, apresentaram menor freqüência de envolvimento positivo e de negativismo aos 30 meses de vida.

A dimensão adaptabilidade esteve negativamente correlacionada ao total dos indicadores de problemas de externalização $(r=-0,51 ; p<0,05)$ e à inadequação $(r=-0,52$; $p<0,01)$, indicando que a adaptação lenta no terceiro mês esteve relacionada tanto a menor freqüência de indicadores de problemas de externalização em geral, como a menor freqüência de inadequação aos 30 meses.

Análise de Regressão Múltipla foi utilizada para verificar a influência de cada uma das seis dimensões do temperamento sobre os indicadores de competência social e de problemas de externalização, mas nenhuma das dimensões do temperamento explicaram esses indicadores do desenvolvimento social.

Por fim, foi encontrada apenas uma correlação positiva entre a responsividade geral da mãe e a categoria de prática educativa materna sensibilidade $(r=0,50 ; p<0,05)$, indicando que quanto maior o nível de responsividade aos três meses do bebê, maior a sensibilidade da mãe aos 30 meses.

Relações entre as Práticas Educativas Maternas e o Comportamento Infantil no $30^{\circ}$ Mês de Vida da Criança

Análises preliminares revelaram várias correlações entre categorias de práticas educativas facilitadoras e os indicadores de competência social e entre as categorias de práticas não-facilitadoras e os indicadores de problemas de externalização (ver Tabela 2). No entanto, ao contrário do

Notas. ${ }^{*} \mathrm{p}<0,05$; ** $\mathrm{p}<0,01$. 
que era esperado, a categoria de comportamento infantil obediência correlacionou-se positivamente com a categoria controle coercitivo, e com o total de práticas não-facilitadoras. Estes resultados sugerem que a obediência, ao contrário dos outros indicadores de competência social avaliados nesse estudo, pode também estar relacionada ao uso de práticas de caráter coercitivo. De modo semelhante, ocorreram também correlações positivas entre algumas categorias de práticas facilitadoras e indicadores dos problemas de externalização. Por exemplo, a desobediência passiva correlacionou-se positivamente com o controle assertivo. Além disso, foram constatadas correlações positivas entre o total de indicadores de problemas de externalização e as categorias orientação e controle assertivo.

O Impacto do Temperamento, da Responsividade e das Práticas Educativas Maternas nos Problemas de Externalização e na Competência Social da Criança no $30^{\circ}$ Mês de Vida

Inicialmente realizou-se uma análise de regressão múltipla para verificar as interações entre o temperamento infantil, a responsividade materna e as práticas educativas maternas facilitadoras, e suas influências sobre a competência social da criança. A Tabela 3 apresenta os resultados desta análise de regressão. Não houve indícios de que o temperamento infantil e a responsividade materna explicassem a competência social da criança. Por outro lado, para cada ocorrência a mais das práticas facilitadoras, a freqüência total de indicadores de competência social da criança aumentou em 0,47; mantendo-se as demais variáveis constantes no modelo $(p=0,001)$. O modelo de regressão múltipla, considerando os três fatores analisados, explicou $36 \%$ ( $R 2$ total ajustado $=0,36$ ) da variância total da competência social das crianças.

Tabela 3

Análise de Regressão Múltipla da Freqüência Total de Indicadores de Competência Social da Criança sobre o Temperamento, a Responsividade e as Práticas Facilitadoras (n=23)

\begin{tabular}{lcccc}
\hline Fatores preditores & $B$ & SE Beta & $\mathrm{p}(T)$ & R2 parcial \\
\hline $\begin{array}{l}\text { Temperamento } \\
\text { infantil }\end{array}$ & 2,23 & 2,87 & 0,77 & 0,17 \\
\hline $\begin{array}{l}\text { Responsividade } \\
\text { materna }\end{array}$ & $-1,68$ & 9,73 & 0,86 & $-0,40$ \\
\hline $\begin{array}{l}\text { Práticas } \\
\text { facilitadoras }\end{array}$ & 0,47 & 0,12 & 0,00 & 0,65 \\
\hline
\end{tabular}

Notas. F=5,20; $\mathrm{p}=0,001$.

Em uma segunda etapa, a análise de regressão múltipla foi empregada para verificar as interações dos três preditores (temperamento, responsividade e práticas) com os indicadores de problemas de externalização da criança. A Tabela 4 apresenta os resultados desta análise de regressão. Não houve indícios de que o temperamento infantil e a responsividade materna explicassem os problemas de externalização. Entretanto, novamente as práticas educativas maternas apareceram como um fator relevante na compreensão desses indicadores. Para cada ocorrência a mais de práticas não-facilitadoras, a freqüência total de indicadores de problemas de externalização da criança aumentou em 0,73 , mantendo-se as demais variáveis constantes no modelo $(p=0,001)$. O modelo de regressão múltipla, considerando os três fatores preditores analisados, explicou $44 \%$ ( $R 2$ total ajustado $=0,44)$ da variância total dos indicadores de problemas de externalização da criança.

Tabela 4

Análise de Regressão Múltipla da Frequiência Total de Indicadores de Problemas de Externalização da Criança sobre o Temperamento a Responsividade e as Práticas Não-Facilitadoras ( $n=23$ )

\begin{tabular}{lcccc}
\hline Fatores preditores & $B$ & SE Beta & $\mathrm{p}(T)$ & R2 parcial \\
\hline Temperamento infantil & $-2,39$ & 1,63 & 0,16 & $-0,31$ \\
Responsividade materna & $-0,95$ & 5,37 & 0,86 & $-0,04$ \\
Práticas não-facilitadoras & 0,73 & 0,16 & 0,00 & 0,70 \\
\hline
\end{tabular}

Notas. $\mathrm{F}=6,93 ; \mathrm{p}=0,001$.

\section{Discussão}

A análise de regressão múltipla dos indicadores de competência social e dos problemas de externalização sobre as seis dimensões do temperamento revelou que nenhuma das dimensões analisadas foi significativa para explicar a variância nesses indicadores. Esses resultados contrariam alguns dos estudos relatados na literatura (Hagekull, 1994; Shaw et al., 2001), mas estão de acordo com os achados de Schaffer (1996) e pela revisão realizada por Prior (1992), que constataram que a maioria das relações encontradas entre temperamento e ajustamento comportamental não se mostravam muito fortes ou consistentes.

Contudo, as análises correlacionais preliminares indicaram relações entre as dimensões intensidade da reação emocional e adaptabilidade e os indicadores de problemas de externalização. Esses resultados indicam a importância da investigação do impacto de dimensões específicas sobre os problemas de externalização e a competência social. Segundo Sanson, Hemphill e Smart (2004), as nove dimensões do temperamento propostas por Thomas e Chess (1977) têm sido amplamente utilizadas em pesquisas. No entanto, alguns estudos que revelaram sobreposição entre as escalas e baixa consistência interna têm exigido refinamentos conceituais, que por sua vez, conduziram a novas dimensões como emocionalidade negativa, aproximação-retraimento e auto-regulação. É possível que as dimensões adaptabilidade e intensidade da reação emocional, utilizadas neste estudo, aproximem-se conceitual e operacionalmente das dimensões emocionalidade negativa e aproximação-retraimento, que têm apresentado relações importantes com características do desenvolvimento social (Sanson et al., 2004). Outra possibilidade é a de que a resposta para essa questão também deva levar em consideração a intersecção entre influências constitucionais e ambientais, as quais têm 
se mostrado até certo ponto indissociáveis (Carey \& McDevitt, 1995).

A responsividade materna geral aos três meses do bebê, foi preditora somente da sensibilidade materna avaliada aos 30 meses da criança, não apresentando relações significativas com as demais práticas avaliadas. A sensibilidade materna foi definida neste estudo como manifestações maternas que indicam atenção, consideração e respeito em relação às necessidades e desejos da criança, quando estes são manifestados de forma assertiva. A categoria foi criada para contemplar práticas descritas na literatura como autorizadoras da autonomia da criança. Os resultados obtidos mostraram que a sensibilidade materna aos sinais do bebê e a capacidade da mãe de responder de forma adequada a esses sinais constituem um importante preditor da capacidade futura da mãe de perceber o crescimento e o desenvolvimento da criança, permitindo-lhe autonomia. Assim, seria interessante pensar em uma sensibilidade que permite à mãe reconhecer a fragilidade e a imaturidade inicial do bebê, que necessita de um cuidador muito presente e altamente contingente, bem como sua necessidade crescente de autonomia à medida que se desenvolve. Desta forma, é possível compreender a relação entre as duas variáveis: nos primeiros meses do bebê a mãe se comportaria de modo a responder prontamente a sinais sutis de desconforto ou tentativas de comunicação, e com o passar do tempo seu comportamento se tornaria "menos intrusivo", estimulando o desenvolvimento da criança para que ela se torne um ser cada vez mais independente, capaz de identificar suas próprias necessidades e desejos e ir em busca de sua satisfação. Assim, os resultados do presente estudo indicam que a responsividade materna pode ser um importante preditor de práticas de autorização da autonomia da criança. Porém, ao contrário dos pressupostos de Patterson et al. (2000), a responsividade parece um conceito insuficiente para contemplar a predição da ampla variedade de práticas educativas facilitadoras e não-facilitadoras do desenvolvimento social da criança.

Quanto à influência das práticas educativas maternas, foram encontradas várias evidências das relações entre as práticas facilitadoras e a competência social, bem como entre as práticas não-facilitadoras e os problemas de externalização. Esses resultados estão de acordo com os inúmeros estudos revisados (Crockenberg \& Litman, 1990; Patterson et al., 1992, 2000) que relacionam positivamente o uso de práticas reforçadoras, assertivas e contingentes ao desenvolvimento social na infância, bem como as práticas coercitivas e não-contingentes, aos problemas de externalização. Além disso, também foram encontradas algumas relações não esperadas entre categorias específicas de práticas facilitadoras e os problemas de externalização e entre certas categorias de práticas não-facilitadoras e a competência social. Estes resultados sugerem que a obediência, por exemplo, ao contrário dos outros indicadores de competência social avaliados nesse estudo, pode ser estimulada por práticas de caráter coercitivo, assim como apóiam a noção de que o uso moderado e consistente desse tipo de prá- tica pode favorecer o desenvolvimento social (Grusec \& Lytton, 1988; Hoffman, 1979). Como os achados a respeito dessas relações foram numerosos, optou-se por explorá-los e discuti-los em outro artigo (Alvarenga \& Piccinini, 2006).

Por fim, é importante assinalar o fato de que o presente estudo foi realizado com uma amostra pequena, o que afetou as análises realizadas. A amostra também apresentava algumas particularidades. Conforme descrito anteriormente, as 23 díades que participaram da pesquisa, constituem uma subamostra de um estudo maior, que acompanhou as famílias desde a gestação do primeiro filho. Ao longo dos três anos em que foram acompanhadas, várias famílias deixaram de participar da pesquisa por diferentes razões. É possível que aquelas que permaneceram ligadas ao estudo apresentassem certas características, que também merecem atenção, como por exemplo, maior interesse e preocupação com o desenvolvimento das crianças e, conseqüentemente, maior envolvimento com seus filhos. Tais características poderiam explicar, em parte, a constatação de alta responsividade materna, da predominância de práticas facilitadoras e, conseqüentemente, da baixa freqüência dos problemas de externalização nas crianças. De qualquer modo, entende-se que estas sejam limitações metodológicas inerentes à realização de um estudo longitudinal que contou com recursos escassos. Por isso, destaca-se a necessidade de que novos estudos investiguem essas questões em amostras maiores.

Em resumo, a hipótese do presente estudo foi parcialmente confirmada. Examinando os resultados das análises de regressão múltipla constatou-se que, embora o temperamento e a responsividade materna não tenham sido significativos para explicar o comportamento infantil, as práticas facilitadoras e não-facilitadoras foram significativas para explicar, respectivamente, a competência social e os problemas de externalização da criança. Diante desses achados, questiona-se a possibilidade de utilizar o temperamento infantil e a responsividade materna como os preditores relevantes das práticas educativas parentais e do comportamento infantil.

\section{Referências}

Achenbach, T. M., \& Howell, C. T. (1993). Are american children's problems getting worse? A 13-year comparision. Journal of American Academiy on Child and Adolescent Psychiatry, 32, 1145-1154.

Alvarenga, P., \& Piccinini, C. A. (2001). Práticas educativas maternas e problemas de comportamento em pré-escolares. Psicologia: Reflexão e Crítica, 14(3), 449-459.

Alvarenga, P., \& Piccinini, C. A. (2006). Relações entre as práticas educativas maternas e indicadores do desenvolvimento social no terceiro ano de vida. Manuscrito não-publicado.

Arsenio, W. F., \& Overton, W. F. (2004). Trajectories of physical aggression from toddlerhood to middle childhood: Predictors, correlates, and outcomes. Monographs of the Society for Research in Child Development, 69(4).

Bolsoni-Silva, A., T., \& Marturano, E. M. (2002). Práticas educativas e problemas de comportamento: Uma análise à luz das habilidades sociais. Estudos de Psicologia, 7(2), 227-235. 
Carey, W. B., \& McDevitt, S. C. (1995). Coping with children's temperament. New York: BasicBooks.

Castro, R. E. F., Melo, M. H. S., \& Silvares, E. F. M. (2003). O julgamento de pares de crianças com dificuldades interativas após um modelo ampliado de intervenção. Psicologia: Reflexão e Crítica, 16(2), 309-318.

Cecconello, A. M., \& Koller, S. H. (2000) Competência social e empatia: Um estudo sobre resiliência com crianças em situação de pobreza. Estudos de Psicologia , 5(1), 71-93.

Cohen, P., \& Brook, J. (1998). The reciprocal influence of punishment and child behavior disorder. In J. McCord (Ed.), Coercion and punishment in long-term perspectives (pp. 154-164). Cambridge, UK: Cambridge University Press.

Crockenberg, S. B., \& Litman, C. (1990). Autonomy as competence in 2-year-olds: Maternal correlates of child defiance, compliance and self-assertion. Developmental Psychology, 26(6), 961-971.

Del Prette, Z. A. P., \& Del Prette, A. (1999). Psicologia das habilidades sociais: Terapia e educação. Petrópolis, RJ: Vozes.

Denham, S. A., Renwick, S. M., \& Holt, R. W. (1991). Working and playing together: Prediction of preschool social-emotional competence from mother-child interaction. Child Development, 62, 242-249.

Denham, S. A., Workman, E., Cole, P. M., Weissbrod, C., Kendisiora, K. T., \& Zahn-Waxler, C. (2000). Prediction of externalizing behavior problems from early to middle childhood: The role of parental socialization and emotion expression. Development and psychopathology, 12, 23-45.

De Wolff, M. S., \& Ijzendoorn, M. H. (1997). Sensitivity and attachment: A meta-analysis on parental antecedents of infant attachment. Child Development, 68(4), 571-591.

Dumas, J. E., \& LaFreniere, P. J. (1993). Mother-child relationships as sources of support or stress: A comparision of competent, average, agressive, and anxious dyads. Child Development, 64, 1732-1754.

Durkin, K. (1995). Developmental social psychology: From infancy to old age. Cambridge, UK: Blackwell.

Eddy, J. M., Leve, L. D., \& Fagot, B. I. (2001). Coercive family processes: A replication and extension of Patterson's coercion model. Agressive Behavior, 27, 14-25.

Falcone, E. O. (2001). Uma proposta de um sistema de classificação das habilidades sociais. In H. J. Guilhardi, M. B. B. P. Madi, P. P. Queiroz, \& M. C. Scoz (Eds.), Sobre comportamento e cognição: Expondo a variabilidade (pp. 195-209). Santo André, SP: ESETec.

Fox, R. A., Platz, D. L., \& Bentley, K. S. (1995). Maternal factors related to parenting practices, developmental expectations, and perceptions of child behavior problems. The Journal of Genetic Psychology, 156, 431-441.

Grupo de Interação Social, Desenvolvimento e Psicopatologia. (1998). Entrevista de dados demográficos do casal. Porto Alegre, RS: Universidade Federal do Rio Grande do Sul. Manuscrito não-publicado.

Grupo de Interação Social, Desenvolvimento e Psicopatologia. (1999a). Observação da interação familiar $-3^{\circ}$ mês. Porto Alegre, RS: Universidade Federal do Rio Grande do Sul. Manuscrito não-publicado.

Grupo de Interação Social, Desenvolvimento e Psicopatologia. (1999b). Entrevista sobre características do temperamento do bebê$3^{o}$ mês. Porto Alegre, RS: Universidade Federal do Rio Grande do Sul. Manuscrito não-publicado.
Grupo de Interação Social, Desenvolvimento e Psicopatologia. (2001). Observação da interação familiar $-30^{\circ}$ mês. Porto Alegre, RS: Universidade Federal do Rio Grande do Sul. Manuscrito não-publicado.

Grusec, J. E., \& Lytton, H. (1988). Social development: History, theory and research. New York: Springer-Verlag.

Guerin, D. W., Gottfried, A., \& Thomas, C. W. (1997). Difficult temperament and behaviour problems: A longitudinal study from 1.5 to 12 years. International Journal of Behaviour Development, 21(1), 71-90.

Hagekull, B. (1994). Infant temperament and early childhood functioning: Possible relations to the five-factor model. In C. J. Halverson, Jr., G. A. Kohnstamm \& R. P. Martin (Eds.), The developing structure of temperament and personality (pp. 227240). Hillsdale, NJ: Erlbaum.

Hoffman, M. L. (1979). Development of moral thought, feeling, and behavior. American Psychologist, 34, 958-966.

Hoffman, M. L. (1994). Discipline and internalization. Developmental Psychology, 30, 26-28.

Hollingshead, A. (1975). The four-factor index of social status. New Haven, CT: Yale University. Unpublished manuscript.

Isabella, R. A., Belsky, J., \& Von Eye, A. (1989). Origins of infant-mother attachment: An examination of interactional synchrony during the infant's first year. Developmental Psychology, 25(1), 12-21.

Keenan, K., \& Shaw, D. (1997). Developmental and social influences on young girls's early problem behavior. Psychological Bulletin, 12 1, 95-1 13.

Keenan, K., \& Shaw, D. (1998). The development of coercive family processes: The interaction between aversive toddler behavior and parenting factors. In J. McCord (Ed.), Coercion and punishment in long-term perspectives (pp. 165-180). Cambridge, UK: Cambridge University Press.

Landry, S. H., Smith, K. E., Swank, P. R., Assel, M. A., \& Vellet, S. (2001). Does early responsive parenting have a special importance for children's development or is consistency across early childhood necessary? Development Psychology, 37(3), 387-403.

Maccoby, E. E. (1966). The development of Sex differences. Stanford, CA: Stanford University Press.

McLoyd, V. C., \& Smith, J. (2002). Phisical discipline and behavior problems in african american, european, and spanic children: Emotional support as a moderator. Journal of Marriage and Family, 64, 40-53.

Oliveira, E. A., Frizzo, G. B., \& Marin, A. H. (2000). Atitudes maternas diferenciais para com meninos e meninas de 4 e 5 anos. Psicologia: Reflexão e Crítica, 13(3), 363-371.

Paley, B., Cox, M. J., \& Kanoy, K. W. (2001). The young family interaction coding system. In P. K. Kerig \& K. M. Lindahl (Eds), Family observational coding systems: Resources for systemic research (pp. 273-288). Mahwah, NJ: Lawrence Erlbaum.

Patterson, G. R., DeGarmo, D. S., \& Knutson, N. (2000). Hyperactive and antisocial behaviors: Comorbid or two points in the same process? Development and Psychopathology, 12, 91-106.

Patterson, G. R., Reid, J., \& Dishion, T. (1992). Antisocial boys. Eugene, OR: Castalia.

Pettit, G. S., Bates, J. E., \& Dodge, K. A. (1997). Supportive parenting, ecological context, and children's adjustment: A seven-year longitudinal study. Child Development, 68, 908- 923.

Piccinini, C. A., Tudge, J. R., Lopes, R. S., \& Sperb, T. M. (1998). Estudo Longitudinal de Porto Alegre: Da gestação à escola. Porto Alegre, RS: Universidade Federal do Rio Grande do Sul, Instituto de Psicologia. Relatório não-publicado. 
Prior, M. (1992). Childhood temperament. Journal of Child Psychology and Psychiatry, 33, 249-280.

Raver, C. C. (1996). Relations between social contingency in mother-child interaction and 2-year-olds' social competence. Developmental Psychology, 32(5), 850-859.

Rothbart, M. K., Ahadi, S. A., Hershey, K. L., \& Fisher, P. (2001). Investigation of temperament at three to seven years: The Children's Behavior Questionnaire. Child Development, 72(5), 1394-1408.

Sanson, A., Hemphill, S., \& Smart, D. (2002). Temperament and social development. In P. K. Smith \& C. H. Hart (Eds.), Handbook of childhood social development (pp. 97-116). London: Blackwell.

Sanson, A., Hemphill, S., \& Smart, D. (2004). Connections between temperament and social development: A review. Social Development, 13, 142-168.

Schaffer, H. R. (1996). Social development. Oxford, UK: Blackwell.

Schwebel, D. C., \&, Plumert, J. M. (1999). Longitudinal and concurrent relations among temperament, ability estimation and injury proneness, Child Development, 70, 700-712.

Seifer, R., Sameroff, A. J., Barrett, L. C., \& Krafchuk, E. (1994). Infant temperament measured by multiple observations and mother report. Child Development, 65, 1478-1490.

Shaw, D. S., Owens, E. B., Giovanelli, J., \& Winslow, E. B. (2001). Infant and toddler pathways leading to early externalizing disorders. Journal of the American Academy of Child and Adolescent Psychiatry 40, 36-43.
Thomas, A., \& Chess, S. (1977). Temperament and development. New York: Pleno.

Tudge, J. R. H., \& Frizzo, G. F. (2002). Classificação baseada em Hollingshead do nível socioeconômico das famílias do estudo longitudinal de Porto Alegre: Da gestação à escola. Manuscrito não-publicado.

van den Boom, D. C. (1994). The influence of temperament and mothering on attachment and exploration: An experimental manipulation of sensitive responsiveness among lower-class mothers with irritable infants. Child Development, 65, 1449-1469.

Weisz, J. R., Chaiyasit, W., Weiss, B., Eastman, K. L., \& Jackson, E. W. (1995). A multimethod study of problem behavior among thai and american children in school: Teacher reports versus direct observations. Child Development, 66, 402-415.

Wendland-Carro, J., Piccinini, C. A., \& Millar, W. S. (1999). The role of an early intervention on enhancing the quality of motherinfant interaction. Child Development, 7O(3), 713-721.

Recebido: 08/06/2006 $1^{a}$ revisão: 14/09/2006 $2^{a}$ revisão: 24/11/2006 Aceite final: 04/12/2006 